\title{
Probability-Dependent Gain-Scheduled Control for Discrete Stochastic Delayed Systems with Randomly Occurring Nonlinearities
}

\author{
Guoliang $\mathrm{Wei}^{a}$, Zidong Wang ${ }^{a, b, *}$ and Bo Shen ${ }^{a}$
}

\begin{abstract}
In this paper, the gain-scheduled control problem is addressed by using probability-dependent Lyapunov functions for a class of discrete-time stochastic delayed systems with randomly occurring sector-nonlinearities. The sectornonlinearities are assumed to occur according to a time-varying Bernoulli distribution with measurable probability in real time. The multiplicative noises are given by means of a scalar Gaussian white noise sequence with known variances. The aim of the addressed gain-scheduled control problem is to design a controller with scheduled gains such that, for the admissible randomly occurring nonlinearities (RONs), time delays and external noise disturbances, the closed-loop system is exponentially mean-square stable. Note that the designed gain-scheduled controller is based on the measured time-varying probability and is therefore less conservative than the conventional controller with constant gains. It is shown that the time-varying controller gains can be derived in terms of the measurable probability by solving a convex optimization problem via the semi-definite programme method. A simulation example is exploited to illustrate the effectiveness of the proposed design procedures.
\end{abstract}

\section{Keywords}

Gain-scheduled control; randomly occurring nonlinearities; time-varying Bernoulli distribution; probability-dependent Lyapunov function; sector-nonlinearity; parameter-varying systems; discrete-time stochastic systems.

\section{INTRODUCTION}

Owing to pervasive existence of stochastic perturbations in reality, stochastic models have been successfully utilized to describe many practical systems such as mechanical systems, economic systems and biological systems, etc. Over the past few decades, the study of stabilization, control and filtering problems for stochastic systems have been paid much attention from many researchers and a large number of results have been reported in the literature, see e.g. $[3,6,11,25,26]$. On the other hand, time delay is usually one of the main sources of poor performance and instability. Therefore, it is not surprising that the stochastic control problem for time-delay systems have received considerable research interests, see $[6,11,14,25-28]$ for some recent results.

As is well known, nonlinearity is often inevitable in many real-world control systems that poses essential challenges for system design. In the past years, the analysis and synthesis problems of nonlinear stochastic systems have constituted the main stream of research in the control community which have been attracting

This work was supported in part by the Leverhulme Trust of the U.K., the Engineering and Physical Sciences Research Council (EPSRC) of the U.K. under Grant GR/S27658/01, the National Natural Science Foundation of China under Grants 61028008, 61134009, 61074016, 61104125 and 60974030, the Shanghai Natural Science Foundation of China under Grant 10ZR1421200, and the Alexander von Humboldt Foundation of Germany.

${ }^{a}$ School of Information Science and Technology, Donghua University, Shanghai 200051, China.

${ }^{b}$ Department of Information Systems and Computing, Brunel University, Uxbridge, Middlesex, UB8 3PH, United Kingdom.

* Corresponding address: School of Information Science and Technology, Donghua University, Shanghai 200051, China. E-mail: Zidong. Wang@brunel.ac.uk 
more and more research attention, see e.g. [26,28]. Among others, the sector-nonlinearity (also called sectorlike nonlinearity) is known to be quite general that includes the widely used Lipschitz condition as a special case. So far, the control, filtering and model reduction problems for systems with sector-nonlinearities have been intensively studied, see e.g. $[12,16-18,26]$ for some recent publications.

Stemming from the popularity of networked control systems where signals are transmitted through channels with limited bandwidth, the randomly occurring phenomenon in system models has started to gain some initial research attention and some important results have been appeared, see e.g. [10, 20, 23, 25, 26, 29]. Roughly speaking, the randomly occurring phenomenon refers to some system complexities (e.g. nonlinearities, uncertainties, information missing, sensor saturations, etc) that occur in a random way based on certain probabilistic law. When modeling the randomly occurring phenomenon especially in the sensor outputs, the Bernoulli distribution model has proven to be a flexible yet effective one that has been frequently employed, see $[10,23,29]$. On the other hand, a large class of nonlinearities results from exogenous disturbances caused by environmental circumstances and may appear intermittently in a random way due probably to random failures and repairs of the components, intermittently switching in the interconnections of subsystems, etc. Such nonlinear disturbances are called randomly occurring nonlinearities (RONs) that have recently introduced in $[22,24]$. It should be pointed out that the RONs in $[22,24]$ have been assumed to satisfy a time-invariant Bernoulli distribution. Such an assumption, unfortunately, limits the application scope since RONs usually appears with time-varying probability. It is, therefore, one of the objectives of this paper to extend the RON model in $[22,24]$ to the time-varying one by pursuing the time-varying gain-scheduled controller design based on the time-varying probability.

On another research frontier, the design problems of gain-scheduled controllers and filters have recently become research focuses attracting persistent research attentions from both control theory and application viewpoints. For some representative literature we refer the readers to $[1,5,6,19]$. In gain-scheduled controllers/filter design, the gains of controllers/filters are time-varying consisting of two parts, one of which is constant matrices that can be obtained by solving a set of linear matrix inequalities (LMIs) or Riccati inequalities, and the other part is the time-varying parameters that is measurable in real time. The controller/filter gains are scheduled along with the time-varying parameters and, therefore, have much less conservatism than the conventional ones with constant (fixed) gains only. The gain-scheduled control/filtering problems have been extensively investigated for both the continuous-time and discrete-time systems in the past decade, see e.g. $[1,7,13,19,30]$. On the other hand, in order to design more robust controllers/filters with less conservatism, the so-called parameter-dependent Lyapunov functions have recently been exploited to cope with uncertain time-varying systems, see e.g. $[2,7,8,15,21]$. Very recently, the parameter-dependent Lyapunov function approach has been applied in the gain-scheduling control/filtering problems so as to achieve better control/filter performance requirements and some results have been reported in the literature $[2,7,21,30]$. However, up to now, these effective techniques have not been used to address the control problems for discrete-time stochastic systems with randomly occurring nonlinearities. It would be interesting to challenge the gain-scheduled control problem with RONs based on time-varying occurring probability, which constitutes the main focus of this paper.

In this paper, we will aim to consider the gain-scheduled state-feedback control problem for the discrete-time stochastic delayed systems with RONs. The main contributions of this paper can be summarized as follows: 1) a new state-feedback control problem is investigated by a gain-scheduling approach for a class of discrete-time stochastic delayed systems with RONs; 2) the nonlinearity disturbances are assumed to occur in a random way 
and a stochastic variable sequence satisfying time-varying Bernoulli distributions is introduced to describe the time-varying feature of the RONs; 3) the time-varying controller gains are affected by not only the constant parameters but also the time-varying probability; 4) an easily implementable algorithm is developed to design the controller with both constant and time-varying parameters. The desired controller is designed by employing the gain-scheduling method which leads to less conservatism than the traditional one with constant gains only. In the simultaneous presence of RONs, time delays and external noise disturbances, the closed-loop system is guaranteed to be exponentially mean-square stable. A simulation example is exploited to illustrate the effectiveness of the proposed design procedures.

The remainder of this paper is organized as follows. In Section II, the gain-scheduled control problem for a class of discrete-time stochastic delayed systems with randomly occurring sector-nonlinearities is formulated. The stability analysis and controller design issues are dealt with in Section III. An example is given in Section IV and the paper is concluded in Section V.

Notation. In this paper, $\mathbb{R}^{n}, \mathbb{R}^{n \times m}, \mathbb{Z}^{+}$denote, respectively, the $n$-dimensional Euclidean space, the set of all $n \times m$ real matrices, the set of all positive integers. $|\cdot|$ refers to the Euclidean norm in $\mathbb{R}^{n}$. I denotes the identity matrix of compatible dimension. The notation $X \geq Y$ (respectively, $X>Y$ ), where $X$ and $Y$ are symmetric matrices, means that $X-Y$ is positive semi-definite (respectively, positive definite). For a matrix $M, M^{T}$ and $M^{-1}$ represent its transpose and inverse, respectively. The shorthand $\operatorname{diag}\left\{M_{1}, M_{2}, \ldots, M_{n}\right\}$ denotes a block diagonal matrix with diagonal blocks being the matrices $M_{1}, M_{2}, \ldots, M_{n}$. In symmetric block matrices, the symbol $*$ is used as an ellipsis for terms induced by symmetry. Matrices, if they are not explicitly stated, are assumed to have compatible dimensions.

\section{Problem Formulation}

Consider the following discrete-time stochastic delayed systems with RONs:

$$
\begin{aligned}
x(k+1) & =A x(k)+A_{d} x(k-d)+B u(k)+\xi(k) C f(z(k))+D x(k) \omega(k) \\
x(0) & =\rho,
\end{aligned}
$$

where $x(k) \in \mathbb{R}^{n}$ is the state, $\rho$ is the initial state of the system, $d$ is a constant delay and $z(k):=G x(k)+$ $G_{d} x(k-d) \cdot \omega(k)$ is an one-dimensional Gaussian white noise sequence satisfying $\mathbb{E}\{\omega(k)\}=0$ and $\mathbb{E}\left\{\omega^{2}(k)\right\}=$ $\sigma^{2} . A, A_{d}, B, C, D, G$ and $G_{d}$ are constant matrices with appropriate dimensions.

The function $f(\cdot)$ with $(f(0)=0)$ represents nonlinear disturbances satisfying the following sector-bounded condition:

$$
\left[f(z(k))-F_{1} z(k)\right]^{T}\left[f(z(k))-F_{2} z(k)\right] \leq 0,
$$

where $F_{1}$ and $F_{2}$ are constant real matrices of appropriate dimensions with $F_{2}-F_{1}>0$. In this case, $f(\cdot)$ is said to belong to the sector $\left[F_{1}, F_{2}\right]$.

For technique convenience, the nonlinear function $f(z(k))$ is decomposed into a linear part and a nonlinear part as

$$
f(z(k))=F_{1} z(k)+f_{s}(z(k)),
$$

and then it follows from (3) that

$$
f_{s}^{T}(z(k))\left(f_{s}(z(k))-F z(k)\right) \leq 0,
$$


where $F=F_{2}-F_{1}>0$.

Remark 1: The sector nonlinearity is more general than the usual Lipschitz condition and has widely been employed to model some nonlinear phenomena in reality, for example, the saturation nonlinearities and nonlinear activation functions in neural networks and gene regulatory networks. In the past few years, the control, filtering and model reduction problems for the systems with sector nonlinearities have been intensively studied, see e.g., $[12,16-18,26]$.

In (1), $\xi(k)$ is a random variable sequence to account for the RONs satisfying the following Bernoulli distribution

$$
\begin{aligned}
& \operatorname{Prob}\{\xi(k)=1\}=\mathbb{E}\{\xi(k)\}=p(k), \\
& \operatorname{Prob}\{\xi(k)=0\}=1-\mathbb{E}\{\xi(k)\}=1-p(k),
\end{aligned}
$$

where $p(k)$ is a time-varying positive scalar sequence that takes values on the interval $\left[p_{1} p_{2}\right] \subseteq\left[\begin{array}{ll}0 & 1\end{array}\right]$ with $p_{1}$ and $p_{2}$ being the lower and upper bounds of $p(k)$, respectively. In this paper, for simplicity, we assume that $\xi(k), \omega(k)$ and $\rho$ are uncorrelated.

Remark 2: The nonlinear disturbances may occur randomly due to some environment reasons and a Bernoulli distribution model has been introduced in $[22,24]$ to describe such randomly occurring phenomenon. However, the Bernoulli distribution employed in the literature has been assumed to be time-invariant. This is certainly conservative to deal with the time-varying systems with RONs according to time-varying probabilities. As such, we are going to utilize a random variable sequence in (6) that satisfies time-varying Bernoulli distributions in order to better describe the randomly intermittent nature of the concerned nonlinear disturbances.

In this paper, we are interested in designing the following gain-scheduled controller

$$
u(k)=K(p) x(k),
$$

where $K(p)$ is the controller gain sequence to be designed that is of the following structure

$$
K(p)=K_{0}+p(k) K_{u},
$$

where, for every time step $k, p(k)$ is the time-varying parameter of the controller gain taking value in $\left[p_{1}, p_{2}\right]$, and $K_{0}, K_{u}$ are the constant parameters of the controller gain to be designed.

Remark 3: The controller gain given in (8) includes not only the constant parameters $K_{0}, K_{u}$ but also the time-varying parameter $p(k)$. Such controller will be scheduled with the time-varying parameter (the timevarying probability in this paper) which would certainly give rise to less conservatism than the conventional controller with constant gains only. Note that gain-scheduled control/filtering problems have recently stirred considerable research attention $[1,5,7,13,19,21]$.

Remark 4: Because of the special structure of the controller gain in (8), the implementation of the gainscheduled controller deserves particular attention. In fact, the implementation consists of the following steps: 1) compute the constant gains $K_{0}$ and $K_{u}$ of the controller by using the main results to be developed in this paper; 2) estimate/measure the time-varying probability $p(k)$ by statistical tests in practice; and 3$)$ obtain the controller gain from (8).

The closed-loop system of (1) with the state-feedback gain-scheduled controller (7) is given as follows:

$$
x(k+1)=A x(k)+A_{d} x(k-d)+\xi(k) C f(z(k))+B K(p) x(k)+D x(k) \omega(k) .
$$

In this paper, our purpose is to design a probability-dependent gain-scheduled controller of the form (7) for the system (1) by exploiting a probability-dependent Lyapunov function and LMI method such that, for all 
admissible time delays, RONS and exogenous stochastic noises, the closed-loop system (9) is exponentially mean-square stable.

\section{MAIN REsults}

The following lemma will be used in the proofs of our main results in this paper.

Lemma 1: (Schur Complement) [4] Given constant matrices $\Sigma_{1}, \Sigma_{2}, \Sigma_{3}$ where $\Sigma_{1}=\Sigma_{1}^{T}$ and $0<\Sigma_{2}=\Sigma_{2}^{T}$. Then $\Sigma_{1}-\Sigma_{3}^{T} \Sigma_{2}^{-1} \Sigma_{3} \geq 0$ if and only if

$$
\left[\begin{array}{cc}
\Sigma_{1} & \Sigma_{3}^{T} \\
\Sigma_{3} & \Sigma_{2}
\end{array}\right] \geq 0 \quad \text { or } \quad\left[\begin{array}{cc}
\Sigma_{2} & \Sigma_{3} \\
\Sigma_{3}^{T} & \Sigma_{1}
\end{array}\right] \geq 0 .
$$

In the following theorem, a probability-dependent gain-scheduled control problem is dealt with for a class of discrete-time stochastic delayed systems (1) with RONs by exploiting Lyapunov theory and convex programming method. A sufficient condition is derived to guarantee the solvability of the desired gain-scheduled control problem and, in the mean time, the parameters of the gain-scheduled controller can be obtained by solving the convex optimization problem via the semi-definite programme method in terms of the measured time-varying probability.

Theorem 1: Consider the discrete-time stochastic delayed systems (9). Assume that there exist positivedefinite matrices $\mathcal{Q}(p(k))>0$ and $\mathcal{Q}_{d}>0$, slack matrix $S$ and matrices $\mathcal{K}_{0}, \mathcal{K}_{u}$ such that the following LMIs hold:

$$
\left[\begin{array}{cccccc}
\mathcal{Q}_{d}-\mathcal{Q}(p(k)) & * & * & * & * & * \\
0 & -\mathcal{Q}_{d} & * & * & * & * \\
F G S & F G_{d} S & -2 I & * & * & * \\
\bar{A} S+B \mathcal{K}(p) & \bar{A}_{d} S & p(k) C & -\Delta_{k+1} & * & * \\
\sigma^{2} D S & 0 & 0 & 0 & -\sigma^{2} \Delta_{k+1} & * \\
\Delta_{p}(k) C F_{1} G S & \Delta_{p}(k) C F_{1} G_{d} S & \Delta_{p}(k) C & 0 & 0 & -\Delta_{p}(k) \Delta_{k+1}
\end{array}\right]<0
$$

where

$$
\begin{aligned}
\bar{A} & =A+p(k) C F_{1} G, \mathcal{K}(p)=\mathcal{K}_{0}+p(k) \mathcal{K}_{u}, \Delta_{p}(k)=p(k)(1-p(k)) \\
\bar{A}_{d} & =A_{d}+p(k) C F_{1} G_{d}, \Delta_{k+1}=-\mathcal{Q}(p(k+1))+S+S^{T}
\end{aligned}
$$

In this case, the constant gains of the desired controller can be obtained as follows

$$
K_{0}=\mathcal{K}_{0} S^{-1}, K_{u}=\mathcal{K}_{u} S^{-1}
$$

and the closed-loops system (9) is then exponentially mean-square stable for all $p(k) \in\left[\begin{array}{ll}p_{1} & p_{2}\end{array}\right]$.

Proof: Let $Q(p(k))=S^{-T} \mathcal{Q}(p(k)) S^{-1}, Q_{d}=S^{-T} \mathcal{Q}_{d} S^{-1}$ and define the following probability-dependent Lyapunov function:

$$
V(k):=x^{T}(k) Q(p(k)) x(k)+\sum_{s=k-d}^{k-1} x^{T}(s) Q_{d} x(s) .
$$


Then, noting $\mathbb{E}\{\xi(k)-p(k)\}=0$ and $\mathbb{E}\{\omega(k)=0\}$, we have from (9) that

$$
\begin{aligned}
& \mathbb{E}\{\Delta V(k)\}=\mathbb{E}\left\{x^{T}(k+1) Q(p(k+1)) x(k+1)-x^{T}(k)\left(Q(p(k))-Q_{d}\right) x(k)\right. \\
&\left.-x^{T}(k-d) Q_{d} x(k-d)\right\} \\
&=\mathbb{E}\{[ A x(k)+A_{d} x(k-d)+p(k) C f(z(k))+(\xi(k)-p(k)) C f(z(k)) \\
&+B K(p) x(k)+D x(k) \omega(k)]^{T} Q(p(k+1))\left[A x(k)+A_{d} x(k-d)\right. \\
&+p(k) C f(z(k))+(\xi(k)-p(k)) C f(z(k))+B K(p) x(k)+D x(k) \omega(k)] \\
&\left.-x^{T}(k)\left(Q(p(k))-Q_{d}\right) x(k)-x^{T}(k-d) Q_{d} x(k-d)\right\} \\
&=\mathbb{E}\{[\left.A x(k)+A_{d} x(k-d)+p(k) C f(z(k))+B K(p) x(k)\right]^{T} Q(p(k+1)) \\
& \times\left[A x(k)+A_{d} x(k-d)+p(k) C f(z(k))+B K(p) x(k)\right] \\
&-x^{T}(k)\left(Q(p(k))-Q_{d}\right) x(k)-x^{T}(k-d) Q_{d} x(k-d) \\
&+p(k)(1-p(k)) f^{T}(z(k)) C^{T} Q(p(k+1)) C f(z(k)) \\
&\left.+\sigma^{2} x^{T}(k) D^{T} Q(p(k+1)) D x(k)\right\} .
\end{aligned}
$$

From (3)-(5) and (14), it can be seen that

$$
\begin{aligned}
\mathbb{E}\{\Delta V(k)\} \leq \mathbb{E}\{[ & \left(A+B K(p)+p(k) C F_{1} G\right) x(k)+\left(A_{d}+p(k) C F_{1} G_{d}\right) x(k-d) \\
& \left.+p(k) C f_{s}(z(k))\right]^{T} Q(p(k+1))\left[\left(A+B K(p)+p(k) C F_{1} G\right) x(k)\right. \\
& \left.+\left(A_{d}+p(k) C F_{1} G_{d}\right) x(k-d)+p(k) C f_{s}(z(k))\right] \\
& \left.+p(k)(1-p(k))\left[f_{s}(z(k))+F_{1} G x(k)+F_{1} G_{d} x(k-d)\right)\right]^{T} C^{T} \\
& \left.\times Q(p(k+1)) C\left[f_{s}(z(k))+F_{1} G x(k)+F_{1} G_{d} x(k-d)\right)\right] \\
& -x^{T}(k)\left(Q(p(k))-Q_{d}\right) x(k)-x^{T}(k-d) Q_{d} x(k-d) \\
& -2 f_{s}^{T}(z(k)) f_{s}(z(k))+\sigma^{2} x^{T}(k) D^{T} Q(p(k+1)) D x(k) \\
& \left.\left.+2 f_{s}^{T}(z(k)) F G x(k)+2 f_{s}^{T}(z(k)) F G_{d} x(k-d)\right)\right\} \\
=\mathbb{E}\{ & \left.\bar{x}^{T}(k) \Omega \bar{x}(k)\right\},
\end{aligned}
$$

where $\bar{x}(k)=\left[\begin{array}{lll}x^{T}(k) & x^{T}(k-d) & f_{s}^{T}(z(k))\end{array}\right]^{T}$ and

$$
\Omega=\left[\begin{array}{ccc}
\Omega_{1} & * & * \\
\Omega_{2} & \Omega_{3} & * \\
\Omega_{4} & \Omega_{5} & \Omega_{6}
\end{array}\right],
$$


with

$$
\begin{aligned}
\Omega_{1}= & {\left[A+B K(p)+p(k) C F_{1} G\right]^{T} Q(p(k+1))\left[A+B K(p)+p(k) C F_{1} G\right]-Q(p(k)) } \\
& +p(k)(1-p(k)) G^{T} F_{1}^{T} C^{T} Q(p(k+1)) C F_{1} G+\sigma^{2} D^{T} Q(p(k+1)) D+Q_{d}, \\
\Omega_{2}= & {\left[A_{d}+p(k) C F_{1} G_{d}\right]^{T} Q(p(k+1))\left[A+B K(p)+p(k) C F_{1} G\right]+p(k)(1-p(k)) } \\
& \times G_{d}^{T} F_{1}^{T} C^{T} Q(p(k+1)) C F_{1} G, \\
\Omega_{3}= & {\left[A_{d}+p(k) C F_{1} G_{d}\right]^{T} Q(p(k+1))\left[A_{d}+p(k) C F_{1} G_{d}\right]+p(k)(1-p(k)) G_{d}^{T} F_{1}^{T} } \\
& \times C^{T} Q(p(k+1)) C F_{1} G_{d}-Q_{d}, \\
\Omega_{4}= & p(k) C^{T} Q(p(k+1))\left[A+B K(p)+p(k) C F_{1} G\right]+p(k)(1-p(k)) C^{T} Q(p(k+1)) \\
& \times C F_{1} G+F G, \\
\Omega_{5}= & p(k) C^{T} Q(p(k+1))\left[A_{d}+p(k) C F_{1} G_{d}\right]+p(k)(1-p(k)) C^{T} Q(p(k+1)) C F_{1} G_{d} \\
& +F G_{d}, \\
\Omega_{6}= & -2 I+p(k) C^{T} Q(p(k+1)) C .
\end{aligned}
$$

In the following, we are going to show that $\Omega<0$ can be concluded from (10). To do this, we pre- and post-multiply the LMIs in (10) by $\operatorname{diag}\left\{S^{-T}, S^{-T}, I, S^{-T}, \sigma^{-2} S^{-T}, \Delta_{p}^{-1}(k) S^{-T}\right\}$ and its transpose, and we can obtain

$$
\left[\begin{array}{cccccc}
Q_{d}-Q(p(k)) & * & * & * & * & * \\
0 & -Q_{d} & * & * & * & * \\
F G & F G_{d} & -2 I & * & * & * \\
S^{-T} \bar{A}+S^{-T} B K(p) & S^{-T} \bar{A}_{d} & p(k) S^{-T} C & -\bar{\Delta}_{k+1} & * & * \\
S^{-T} D & 0 & 0 & 0 & -\sigma^{-2} \bar{\Delta}_{k+1} & * \\
S^{-T} C F_{1} G & S^{-T} C F_{1} G_{d} & S^{-T} C & 0 & 0 & -\Delta_{p}^{-1}(k) \bar{\Delta}_{k+1}
\end{array}\right]<0
$$

with $\bar{\Delta}_{k+1}=-Q(p(k+1))+S^{-1}+S^{-T}$.

Let us now show that, if (18) holds, then the LMIs

$$
\left[\begin{array}{cccccc}
Q_{d}-Q(p(k)) & * & * & * & * & * \\
0 & -Q_{d} & * & * & * & * \\
F G & F G_{d} & -2 I & * & * & * \\
\bar{A}+B K(p) & \bar{A}_{d} & p(k) C & -\Pi_{k+1} & * & * \\
D & 0 & 0 & 0 & -\sigma^{-2} \Pi_{k+1} & * \\
C F_{1} G & C F_{1} G_{d} & C & 0 & 0 & -\Delta_{p}^{-1}(k) \Pi_{k+1}
\end{array}\right]<0
$$

where $\Pi_{k+1}=Q^{-1}(p(k+1))$, are true.

If (18) holds, we can know from $-Q(p(k+1))+S^{-1}+S^{-T}>0$ that $S^{-1}$ is a nonsingular matrix. By performing the congruence transformation $\operatorname{diag}\{I, I, I, S, S, S\}$ to (18), we can have

$$
\left[\begin{array}{cccccc}
Q_{d}-Q(p(k)) & * & * & * & * & * \\
0 & -Q_{d} & * & * & * & * \\
F G & F G_{d} & -2 I & * & * & * \\
\bar{A}+B K(p) & \bar{A}_{d} & p(k) C & -\Gamma_{k+1} & * & * \\
D & 0 & 0 & 0 & -\sigma^{-2} \Gamma_{k+1} & * \\
C F_{1} G & C F_{1} G_{d} & C & 0 & 0 & -\Delta_{p}^{-1}(k) \Gamma_{k+1}
\end{array}\right]<0
$$


with $\Gamma_{k+1}=-S^{T} Q(p(k+1)) S+S+S^{T}$. Then, (19) can be inferred from the inequality $Q^{-1}(p(k+1)) \geq$ $-S^{T} Q(p(k+1)) S+S+S^{T}$. Furthermore, by Lemma 1, we can know from (19) that $\Omega<0$ and, subsequently,

$$
\mathbb{E}\{\Delta V(k)\}<-\lambda_{\min }(\Omega) \mathbb{E}|\bar{x}(k)|^{2}
$$

where $\lambda_{\min }(\Omega)$ is the minimum eigenvalue of $\Omega$. Finally, it can be confirmed from Lemma 1 of [23] that the closed-loop system (9) is exponentially mean-square stable. The proof of this theorem is now complete.

Remark 5: In Theorem 1, in order to obtain desired controller gains by making use of the time-varying probability, probability-dependent Lyapunov functions have been exploited to reduce the conservatism of controller design. Note that, in the past few years, parameter-dependent Lyapunov functions have been often applied to uncertain systems and time-varying parameter systems [7,8,21]. Also, a slack variable $S$ has been introduced In Theorem 1 to decouple the product terms between Lyapunov matrices and system matrices in order that we can easily deal with the resulting controller design problem. Such a technique has been extensively utilized to design controllers/filters $[2,5,8,9,13,30]$.

Remark 6: In Theorem 1, the constant parameters of the controller gain $K_{0}$ and $K_{u}$ can be obtained by solving the LMIs in (10) and, therefore, the gain-scheduled controller gain can be derived by using the measured time-varying probability in real time. However, (10) are LMIs dependent on $p(k) \in\left[p_{1} p_{2}\right]$, so the number of LMIs in (10) is actually infinite. In this case, the desired controller cannot be obtained directly from Theorem 1 due to the infinite number of LMIs. To handle such a problem, in the next theorem, we attempt to convert the problem into a computationally accessible one by changing the description of $p(k)$.

To deal with the computational issue, we will assign a specific form to $p(k)$. Let us set $Q(p(k))=Q_{0}+p(k) Q_{p}$ and we can easily know that $\mathcal{Q}(p(k))=\mathcal{Q}_{0}+p(k) \mathcal{Q}_{p}$.

Theorem 2: Consider the discrete-time nonlinear stochastic systems (1). If there exist positive-definite matrices $\mathcal{Q}_{0}>0, \mathcal{Q}_{p}>0$ and $\mathcal{Q}_{d}>0$, matrices $S, \mathcal{K}_{0}$ and $\mathcal{K}_{u}$ such that the following LMIs hold:

$$
\mathbb{M}^{i j l}=\left[\begin{array}{ccccccc}
\mathcal{Q}_{d}-\mathcal{Q}_{0}-p_{i} \mathcal{Q}_{p} & * & * & * & * & * & \\
0 & -\mathcal{Q}_{d} & * & * & * & * & \\
F G S & F G_{d} S & -2 I & * & * & * & * \\
\tilde{A}^{i j l} & \tilde{A}_{d}^{i j l} & p_{i} C & -\Delta^{i j l} & * & * & * \\
\sigma^{2} D S & 0 & 0 & 0 & 0 & -\sigma^{2} \Delta^{i j l} & \\
\Lambda^{i j l} C F_{1} G S & \Lambda^{i j l} C F_{1} G_{d} S & \Lambda^{i j l} C & 0 & 0 & -\Lambda^{i j l} \Delta^{i j l} & *
\end{array}\right]<0, i, j, l=1,2,
$$

where

$$
\begin{aligned}
\tilde{A}^{i j l} & =A S+B \mathcal{K}_{0}+p_{i} B \mathcal{K}_{u}+p_{i} C F_{1} G S, \\
\tilde{A}_{d}^{i j l} & =A_{d} S+p_{i} C F_{1} G_{d} S, \Lambda^{i j l}=p_{i}\left(1-p_{j}\right), \\
\Delta^{i j l} & =-\mathcal{Q}_{0}-p_{l} \mathcal{Q}_{p}+S+S^{T},
\end{aligned}
$$

and $\mathcal{K}_{0}, \mathcal{K}_{u}$ have been defined in (12), then there exists a controller in the form of (7) such that the closed loop system (9) is exponentially mean-square stable.

Proof: First, letting

$$
\alpha_{1}(k)=\frac{p_{2}-p(k)}{p_{2}-p_{1}}, \quad \alpha_{2}(k)=\frac{p(k)-p_{1}}{p_{2}-p_{1}},
$$

we have

$$
p(k)=\alpha_{1}(k) p_{1}+\alpha_{2}(k) p_{2}
$$


with $\alpha_{i}(k) \geq 0(i=1,2)$ and $\alpha_{1}(k)+\alpha_{2}(k)=1$.

Similarly, letting

$$
\beta_{1}(k)=\frac{p_{2}-p(k+1)}{p_{2}-p_{1}}, \quad \beta_{2}(k)=\frac{p(k+1)-p_{1}}{p_{2}-p_{1}},
$$

it can be obtained that

$$
p(k+1)=\beta_{1}(k) p_{1}+\beta_{2}(k) p_{2}
$$

where $\beta_{i}(k) \geq 0(i=1,2), \beta_{1}(k)+\beta_{2}(k)=1$.

By means of the transformations defined above, it can be easily derived that

$$
\mathcal{Q}(p(k))=\mathcal{Q}_{0}+\sum_{i=1}^{2} \alpha_{i}(k) p_{i} \mathcal{Q}_{p}, \quad \mathcal{K}(p)=\mathcal{K}_{0}+\sum_{i=1}^{2} \alpha_{i}(k) p_{i} \mathcal{K}_{u}, \quad \mathcal{Q}(p(k+1))=\mathcal{Q}_{0}+\sum_{l=1}^{2} \beta_{l}(k) p_{l} \mathcal{Q}_{p} .
$$

On the other hand, it is easy to deduce from (22) that

$$
\sum_{i, j, l=1}^{2} \alpha_{i}(k) \alpha_{j}(k) \beta_{l}(k) \mathbb{M}^{i j l}<0 .
$$

From (24)-(27), it can be concluded that (10) is true. The proof is now complete.

Remark 7: In Theorem 2, the infinite LMIs that are dependent on the time-varying probability in Theorem 1 have been changed to finite ones that are dependent on the upper and lower bound of $p(k)$. By Theorem 2 , we can easily obtain the constant parameters of controller by solving a set of LMIs via Matlab LMI toolbox.

In the following, we outline the design procedure given in Theorem 2 as a computationally appealing gainscheduled control algorithm.

Algorithm 1: Discrete-time gain-scheduled controller design algorithm.

Step 1: Given the initial values for the positive integer $N$, the initial state $\rho$, time delay $d, p_{1}$ and $p_{2}$, the matrices $A, A_{d}, B, C, D, F_{1}, F_{2}, G$ and $G_{d}$. Set $k=0$.

Step 2: Solve the linear matrix inequalities in (22) for $i, j, l=1,2$ to obtain the positive-definite matrices $\mathcal{Q}_{0}, \mathcal{Q}_{p}, \mathcal{Q}_{d}$, matrices $S, \mathcal{K}_{0}, \mathcal{K}_{u}$ and then the constant parameters $K_{0}, K_{u}$ by (12).

Step 3: From measured time-varying probability $p(k)$ in real time and (8), derive gain-scheduled controller gain $K(p)$ and then set $k=k+1$.

Step 4: If $k<N$, then go to Step 3, otherwise go to Step 5.

Step 5: Stop.

\section{An Illustrative Example}

In this section, the gain-scheduled controller is designed for the discrete-time stochastic delayed systems with randomly occurring nonlinearities.

The system parameters are given as follows:

$$
\begin{gathered}
A=\left[\begin{array}{cc}
0.87 & 0.2 \\
0 & 0.955
\end{array}\right], A_{d}=\left[\begin{array}{cc}
0.23 & 0.2 \\
0 & 0.33
\end{array}\right], B=\left[\begin{array}{cc}
0.16 & 0 \\
0 & 0.46
\end{array}\right], \\
C=\left[\begin{array}{cc}
0.1 & 0 \\
0 & 0.2
\end{array}\right], D=\left[\begin{array}{cc}
0.23 & 0 \\
0.15 & 0.18
\end{array}\right], F_{1}=\left[\begin{array}{cc}
0.06 & 0 \\
0 & 0.07
\end{array}\right], \\
F_{2}=\left[\begin{array}{cc}
4.81 & 0 \\
0 & 2.95
\end{array}\right], G=\left[\begin{array}{cc}
0.51 & 0 \\
0 & 0.621
\end{array}\right], G_{d}=\left[\begin{array}{cc}
0.31 & 0 \\
0 & 0.22
\end{array}\right], \\
p_{1}=0.39, p_{2}=0.91, \sigma^{2}=1 .
\end{gathered}
$$




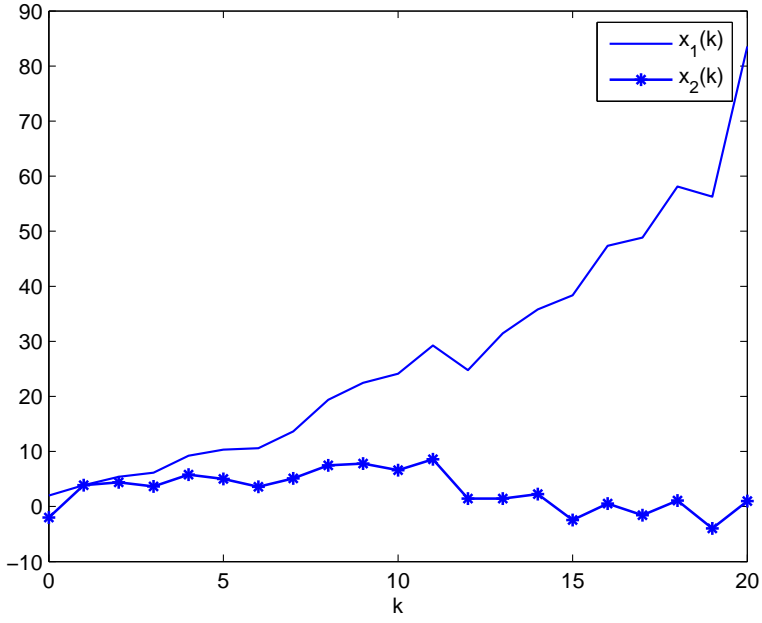

Fig. 1. State evolution $x(k)$ of uncontrolled systems

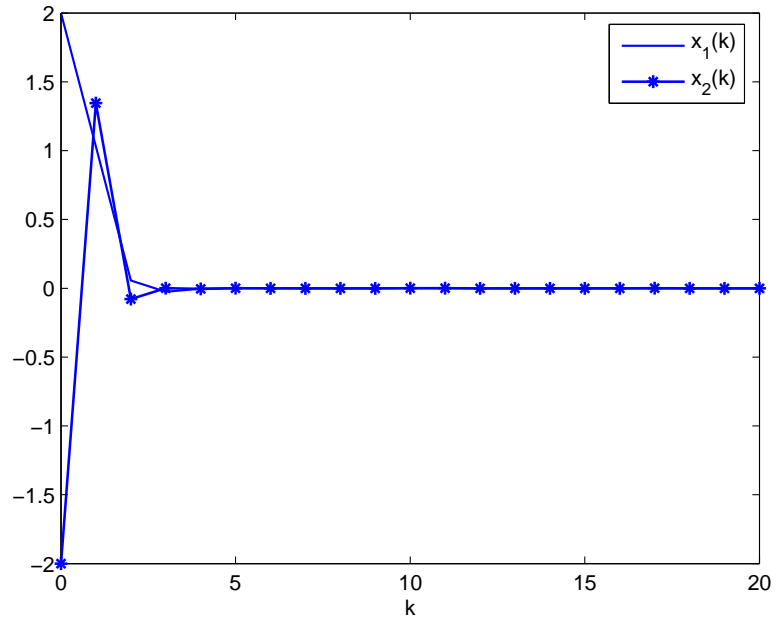

Fig. 2. State evolution $x(k)$ of controlled systems

Set the time-varying Bernoulli distribution sequences as $p(k)=p_{1}+\left(p_{2}-p_{1}\right)|\sin (k)|$ and the sector nonlinear function $f(u)$ as

$$
f(u)=\frac{F_{1}+F_{2}}{2} u+\frac{F_{2}-F_{1}}{2} \sin (u)
$$

which satisfies (3). Also, select the initial state as $\rho=\left[\begin{array}{ll}2 & -2\end{array}\right]^{T}$.

According to Theorem 2 and Algorithm 1, the constant controller parameters $K_{0}, K_{u}$ can be obtained as follows:

$$
\begin{gathered}
Q_{0}=\left[\begin{array}{cc}
10.1578 & 0.4002 \\
0.4002 & 4.5863
\end{array}\right], Q_{p}=\left[\begin{array}{cc}
0.0791 & -0.0086 \\
-0.0086 & 0.0698
\end{array}\right], Q_{d}=\left[\begin{array}{cc}
4.1365 & 0.4313 \\
0.4313 & 2.0879
\end{array}\right], \\
K_{0}=\left[\begin{array}{cc}
-3.7662 & -0.9641 \\
0.1053 & -2.2874
\end{array}\right], K_{u}=\left[\begin{array}{cc}
-0.6804 & 0.0041 \\
0.0287 & -0.4942
\end{array}\right] .
\end{gathered}
$$

Then, the gain-scheduled controller gain $K(p)$ and parameter-dependent Lyapunov matrix can be calculated at every time step $k$ as in Table I.

\begin{tabular}{|c|c|c|c|c|c|c|c|c|c|}
\hline$k$ & \multicolumn{2}{|c|}{0} & \multicolumn{2}{|c|}{1} & \multicolumn{2}{|c|}{2} & \multicolumn{2}{|c|}{3} & $\cdots$ \\
\hline$p(k)$ & \multicolumn{2}{|c|}{0.8276} & \multicolumn{2}{|c|}{0.8628} & \multicolumn{2}{|c|}{0.4634} & \multicolumn{2}{|c|}{0.7835} & . \\
\hline \multirow{2}{*}{$Q(p(k))$} & {$[10.223$} & $0.3931]$ & {$[10.226$} & 0.3928 & 10.194 & $0.3962]$ & {$[10.2198$} & $0.3935]$ & \\
\hline & 0.393 & $4.6441]$ & 0.3928 & $4.6466]$ & 0.3962 & 4.6186 & 0.3935 & $4.6410]$ & \\
\hline \multirow{2}{*}{$K(p)$} & -4.329 & -0.9607 & -4.3533 & -0.9605 & -4.0815 & -0.9622 & -4.2994 & -0.9609 & \\
\hline & 0.1290 & -2.6964 & 0.1300 & -2.7138 & 0.1186 & -2.5164 & 0.1278 & -2.6746 & \\
\hline
\end{tabular}

\section{TABLE I}

\section{Computing Results}

Fig. 1 gives the response curves of state $x(k)$ of uncontrolled systems. Fig. 2 depicts the simulation results of state $x(k)$ of the controlled systems. Fig. 3 shows the time-varying probability parameters $p(k)$. The simulation results have illustrated our theoretical analysis. 


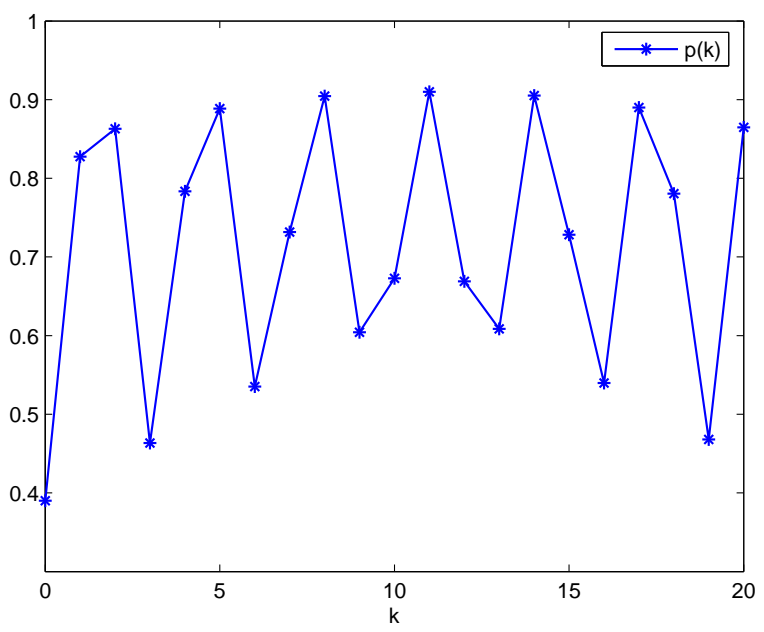

Fig. 3. Time-varying probability $p(k)$

\section{Conclusions}

This paper has dealt with the probability-dependent gain-scheduled state feedback control problem for a class of discrete-time stochastic delayed systems with randomly occurring nonlinearities. We assume the nonlinear disturbances to be randomly occurring and the occurring way is modeled by a stochastic variable sequence satisfying time-varying Bernoulli distributions. By employing probability-dependent Lyapunov functions, we have designed a gain-scheduled controller with the gain including both constant parameters and time-varying parameters such that, for the admissible RONs, time delays and external noise disturbances, the closed-loop system is exponentially mean-square stable. The effectiveness of the proposed design procedure has been illustrated via a numerical example. Moreover, we can extend the main results in this paper to more complex and realistic systems such as systems with polytopic or norm-bounded uncertainties. On the other hand, we will also consider the corresponding filter design problem for stochastic systems with randomly occurring nonlinearities as well as the real-time applications in network-based communications and bioinformatics.

\section{REFERENCES}

[1] P. Apkarian and P. Gahinet, A convex characterization of gain-scheduled $H_{\infty}$ controllers, IEEE Transactions on Automatic Control, Vol. 40, No. 5, pp. 853-864, 1995.

[2] P. Apkarian, P. Pellanda and H. Tuan, Mixed $H_{2} / H_{\infty}$ multi-channel linear parameter-varying control in discrete time, Systems 8 Control Letters, Vol. 41, pp. 333-346, 2000

[3] E. K. Boukas and Z. K. Liu, Deterministic and stochastic time delay systems, Birkhauser, Boston, 2002.

[4] S. Boyd, L. EI Ghaoui, E. Feron and V. Balakrishnan, Linear Matrix Inequalities in Systems and Control Theory, SIAM, Philadelphia, PA, 1994.

[5] Y. Y. Cao, Z. Lin and Y. Shamash, Set invariance analysis and gain-scheduling control for LPV systems subject to actuator saturation, Systems $\&$ Control Letters, Vol. 46, pp. 137-151, 2002.

[6] W. Chen, Z. Guan and X. Liu, Delay-dependent exponential stability of uncertain stochastic systems with mutiple delays: an LMI approach, Systems $\&$ Control Letters, Vol. 54, No. 6, pp. 547-555, 2005.

[7] C. E. de Souza and A. Trofino, Gain-scheduled $H_{2}$ controller synthesis for linear parameter varying systems via parameterdependent Lyapunov functions, International Journal Robust Nonlinear Control, Vol. 16, pp. 243-257, 2006.

[8] H. Gao, P. Shi and J. Wang, Parameter-dependent robust stability of uncertain time-delay systems, Journal of Computational and Applied Mathematics, Vol. 206, pp. 366-373, 2007. 
[9] H. Gao and C. Wang, A delay-dependent approach to robust $H_{\infty}$ filtering for uncertain discrete-time state-delayed systems, IEEE Transactions on Signal Processing, Vol. 52, No. 6, pp. 1631-1640, 2004.

[10] H. Gao, and T. Chen, $H_{\infty}$ estimation for uncertain systems with limited communication capacity, IEEE Trans. Automatic Control. Vol. 52, No. 11, pp. 2070-2084, 2007.

[11] H. Gao, J. Lam and C. Wang, Robust energy-to-peak filter design for stochastic time-delay systems, Systems E Control Letters, Vol. 55, No. 2, pp. 101-111, 2006.

[12] Q. Han, Absolute stability of time-delay systems with sector-bounded nonlinearity, Automatica, Vol. 41, pp. 2171-2176, 2005.

[13] N. Hoang, H. Tuan, P. Apkarian and S. Hosoe, Gain-Scheduled Filtering for Time-Varying Discrete Systems, IEEE Transactions on Signal Processing, Vol. 52, No. 9, 2004, pp. 2464-2476.

[14] H. R. Karimi, Robust delay-dependent $H_{\infty}$ control of uncertain time-delay systems with mixed neutral, discrete, and distributed time-delays and Markovian switching parameters, IEEE Transactions on Circuits and Systems I: Regular Papers, Vol. 58, No. 8, pp. 1910-1923, 2011.

[15] H. R. Karimi, P. Jabedar Maralani, B. Lohmann, and B. Moshiri, $H_{\infty}$ control of parameter-dependent state-delayed systems using polynomial parameter-dependent quadratic functions, International Journal of Control, Vol. 78, No. 4, pp. 254-263, 2005 .

[16] S. J. Kim and I. J. Ha, A state-space approach to analysis of almost periodic nonlinear systems with sector nonlinearities, IEEE Trans. Automat. Control, Vol. 44, No. 1, pp. 66-70, 1999.

[17] J. Lam, H. Gao, S. Xu and C. Wang, $H_{\infty}$ and $L_{2} / L_{\infty}$ model reduction for system input with sector nonlinearities, J. Optimization Theory and Applications, Vol. 125, No. 1, pp. 137-155, 2005.

[18] Z. Lin and T. Hu, Semi-global stabilization of linear systems subject to output saturation, Systems and Control letters, Vol. 43, pp. 211-217, 2001.

[19] W. J. Rugh and J. S. Shamma, Research on gain scheduling, Automatica, Vol. 36, pp. 1401-1425, 2000.

[20] Z. Shu, J. Lam and J. Xiong, Non-fragile exponential stability assignment of discrete-time linear systems with missing data in actuators, IEEE Trans. Automatic Control. Vol. 54, No. 3, pp. 625-630, 2009.

[21] F. Wang and V. Balakrishnan, Improved stability analysis and gain-scheduled controller synthesis for parameter-dependent systems, IEEE Transactions on Automatic Control, Vol. 47, No. 5, pp. 720-734, 2002.

[22] Y. Wang, Z. Wang and J. Liang, Global synchronization for delayed complex networks with randomly ocurred nonlinearities and multiple stochastic disturbances, J. Physics A: Mathematical and Theoretical, Vol. 42, No. 13, 2009 , art. No. 135101.

[23] Z. Wang, F. Yang, D.W.C. Ho, and X. Liu, Robust $H_{\infty}$ filtering for stochastic time-delay systems with missing measurements, IEEE Trans. on Signal Processing, Vol. 54, No. 7, pp. 2579-2587, 2006.

[24] Z. Wang, Y. Wang and Y. Liu, Global synchronization for discrete-time stochastic complex networks with randomly occurred nonlinearities and mixed time-delays, IEEE Transactions on Neural Networks, Vol. 21, No. 1, pp. 11-25, 2010.

[25] G. Wei, Z. Wang and H. Shu, Robust filtering with stochastic nonlinearities and multiple missing measurements, Automatica, Vol. 45, No. 3, pp. 836-841, 2009.

[26] G. Wei, Z. Wang, X. He and H. Shu, Filtering for networked stochastic time-delay systems with sector nonlinearity, IEEE Trans. on Circuits and systems II: Express Briefs, Vol. 56, No. 1, pp. 71-75, 2009.

[27] S. Xu, J. Lam, X. Mao, Y. Zou, A new LMI condition for delay-dependent robust stability of stochastic time-delay systems, Asian Journal of Control, Vol. 7, No. 4, pp. 419-423, 2005.

[28] D. Yue and Q. Han, Dleay-dependent exponential stability of stochastic systems with time-varing delay, nonlinearity and Markovian switching, IEEE Trans. Automatic Control, Vol. 50, No. 2, pp. 217-222, 2005.

[29] F. Yang, Z. Wang, D. W. C. Ho and M. Gani, Robust $H_{\infty}$ control with missing measurements and time-delays, IEEE Trans. Automat. Control, Vol. 52, No. 9, pp. 1666-1672, 2007.

[30] S. Zhou, B. Zhang, and W. Zheng, Gain-scheduled $H_{\infty}$ filtering of parameter-varying discrete-time systems via parameterdependent Lyapunov functions, International Journal of Control, Automation, and Systems, Vol. 7, No. 3, pp. 475-479, 2009 . 